\title{
Different Brainstorming Methods Within the Context of Physical Computing
}

\author{
Alexander Wiethoff, Raphael Wimmer, Hendrik Richter, Andreas Butz \\ Media Informatics Group, University of Munich \\ Amalienstr. 17 \\ 80333 Munich, Germany \\ firstname.lastname@ifi.lmu.de
}
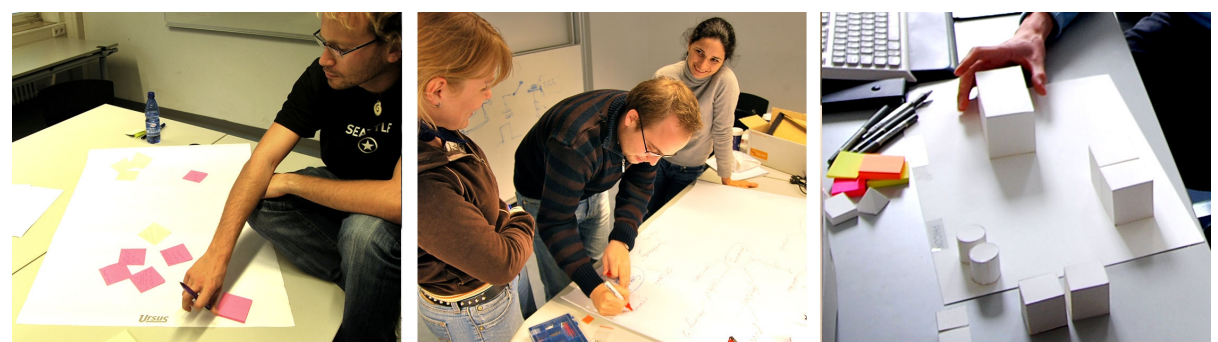

Fig. 1. Three different brainstorming techniques employed in our course (from left to right): Post-Its $^{\circledR}$, creative thinking and tangible 3D primitives

\begin{abstract}
In this work, we want to present a teachable moment in the context of a physical computing and prototyping course held at our university. We tried to investigate how different brainstorming methods affected the ideation phase of a project. To do this, we conducted an experiment in which we separated teams and gave them three different ideation methods: Post-Its ${ }^{\circledR}$, creative thinking (mind mapping) and a physical brainstorming set consisting of cardboard objects to generate their concepts. The conclusions drawn from these observations encouraged us to open up the domain for further experimental setups to investigate the question: Do opportunistic techniques provide a benefit in this context?
\end{abstract}

Keywords: brainstorming, physical computing, design process, tangible user interfaces (TUIs)

\section{INTRODUCTION}

Physical computing and experience prototyping [3,5] are essential tasks as they are tools in the design process to convince others and measure early evaluation. In the course "Sketching with Hardware", taught at our university, students gain knowledge on how to build their own prototypes with a small prototyping platform called Arduino [7]. This environment provides software and hardware to easily establish connections between various sensors and multimedia computers. Within this course, we wanted to know if interaction types such as tangible user interfaces (TUIs) demanded new brainstorming methods in the ideation phase. Much research has focused on the problem of creativity loss in the ideation phase in usercentered design processes, [9] but these days only a few methods are used for ideation. An interesting low-fidelity approach, where office materials such as scissors, paper, pens and glue, is described by Svanæs et al. [10]: Post-Its ${ }^{\circledR}$ serve as a brainstorming mediator. Participants collect ideas on individual Post-Its ${ }^{\circledR}$, cluster them and vote silently for the best ideas. In their workshop participants were building mock-ups of their ideas and stuck Post-Its ${ }^{\circledR}$ on foam-core to simulate early prototypes. Another popular method is creative thinking as described by Cropley, [4] where umbrella terms are placed in the center and associations are arranged around them in bubbles. Given that the goal of such brainstorming sessions is to envision physical, three-dimensional (3D) objects, it seems straightforward to support the brainstorming process with physical 3D tools. However, there are very few studies on how physical 3D objects can stimulate the concept generation phase in this context such as described by $[1,6,8]$. These research projects proposed augmented environments and objects to foster creativity, and we wanted to know if an approach using simple geometrical low fidelity forms provided a benefit, based on Austin [2], who described the need for alternative stimuli. In the following, we describe an initial experiment focusing on two questions: 1) How can different brainstorming methods affect the teams' communication? 2) Can physical, 3D low-fidelity artifacts stimulate the ideation phase better than conventional ideation techniques for developing a TUI?

\section{InITIAL STUDY}

\section{A. Setup}

In the 2009 winter term, we held a course "Sketching with Hardware". Over the course of a week, eight students learned how to design, manufacture and program interactive TUIs. In the brainstorming phase of the course, we organized the students into multidisciplinary teams of 2-3 students. Each team consisted of a media informatics student and a multimedia and art student. These teams 

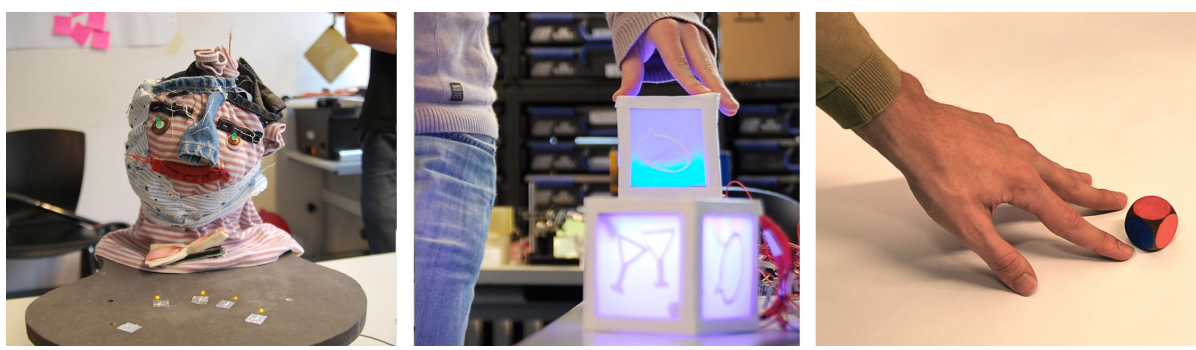

Fig. 2. (From left) Final prototypes: (a) learning puppets for kids, (b) communication cubes for ambient status broadcasting and (c) shuffle remote for controlling an audio player by turning a colored dice.

were paired individually by the course instructors. None of the team members knew each other. They were briefed to brainstorm on a topic in the area of physical computing which could include, but need not be limited to, the keyword "playful". The teams were told to use one specific brainstorming method. The first team was briefed to use Post- $I t^{\circledR}$ notes and a wall to collect and cluster them within different umbrella topics. The second team was instructed to use the method of creative writing and sketch umbrella topics on a big sheet of paper while they quickly spanned associations in "thought bubbles" around these topics. The third team was provided with $153 \mathrm{D}$ primitives made of cardboard, consisting of cubes, cylinders, pyramids and spheres. This team was instructed to use these objects as a brainstorming facilitator and to write down ideas they had in a notebook. After a brainstorming phase of exactly 45 minutes, every team had to vote for their best ideas silently, and each team member had three votes. While the brainstorming sessions were in progress, the course instructors were silently observing every room with the help of co-workers. Two hundred images were taken and observations written down regarding three questions inspired from the scoring test of creative thinking that Cropley illustrated [4]: How much communication was happening? How high was the creative output, regarding quantity(fluency), flexibility (variability) and originality (uncommonness) of the ideas? How independently were the teams working in this phase? The students were not told that they were part of a study or experimental setup to avoid bias.

\section{B. Observations during the Brainstorming Phase}

The first team using the Post-It ${ }^{\circledR}$ notes had initial problems sketching their ideas as the notes were limiting the process due to their shape and size. The team members were working very independently and produced many ideas (high fluency) but had some issues deciding on a direction for a concept they wanted to pursue and got stuck in one area (low variability). Also, the organization of their ideas was rather chaotic; for example, in the beginning they had trouble identifying what they considered to be an "idea" and what was just a good thought. The second team, using the creative thinking method, had the most difficulties in getting the communication flow started (low fluency). After some suggestions, they were unsure about how to continue. We had to restart the process several times and helped by making suggestions and comments on the next steps. When they had to vote for their best ideas, it was hard for them to deliver judgments on the quality of the ideas (low uncommonness). The voting was rather hardware-driven, i.e., the students judged which technologies or sensors they wanted to explore. The third team, using the physical objects, were very quick in deciding on a specific idea and they then focused on details, metaphors and variations early on (high fluency), when the other teams were still very broad in their scope. We noticed that the third team lacked a big variety of ideas (low flexibility), but they still presented very detailed design concepts on that limited number of ideas at the end of the session. To quote one student when discovering the objects (translated from the German): "We were very happy that a series of objects were already there and we could use them to "play and act out' our ideas with them." We also observed that in the third team the physical prototype provided a basis for highly dynamic interpersonal communications.

\section{Resulting Prototypes}

In the final presentation, the team using Post-Its ${ }^{\circledR}$ presented an interactive learning toy for kids. A puppet head nods or shakes and flashes its LED eyes, depending on whether an algebra question is answered correctly or not. The question (e.g., 2+2=?) is presented on a small LCD. Five conductive pads in front of the head are connected to a QProx capacitive sensing IC. The user answers the question by pressing the according number of fingers onto the pads (Figure 2a). The prototype presented by the team using the creative thinking method is named "communication blocks". It consists of three individual cubes. The cubes communicate the user's status in an ambient way through color changes and wireless communication. Modes like drink-ordering, rendezvous or party can be activated by rotating, connecting and stacking the cubes (Figure 2b). The team brainstorming with the physical primitives presented a working prototype of a colored dice as a remote control for an MP3 player. Throwing the dice shuffles the playlist, a tilting switches to the next or previous song, and turning the object changes the volume. In a cooking or kitchen scenario or in other places where tidiness is an issue, this little remote control could provide wireless help while the user could continue his or her task without being worried about damaging the music device (Figure 2c). 


\section{Discussion}

Looking at the way the three brainstorming methods shaped the brainstorming process and the final prototypes, we noted two things. We think that all three methods produced interesting ideas. However, the team using the least tangible brainstorming method-creative thinkinghad much more trouble finding an interesting concept than the other two teams. The team using the $3 \mathrm{D}$ primitives quickly focused on one concept. Therefore, we suggest that tangible brainstorming tools might speed up the ideation process and make users focus on one concept more quickly. On the other hand, the choice of the brainstorming method seemed to have very little impact on the physical quality of the final prototype.

Quite to the contrary, the group using 3D primitives developed the most conservative concept, while the group applying creative writing produced the most elaborate and playful interface. Therefore, we suggest that abstract brainstorming methods like creative thinking are better suited for thinking about interaction concepts than concrete tools like 3D primitives.

In summary, our experiment suggests that tangible brainstorming tools might speed up the ideation process at the cost of reducing the design space. Focusing on the physical properties of an interactive prototype might distract from the interaction concept. On the other hand, tangible prototyping tools might be a useful catalyst for groups that are not yet familiar with more abstract ideation methods. As we had only one group using each method, these findings might be due to chance.

\section{Future Work}

The initial findings described above seem worthwhile to investigate further. We are currently planning a new "Sketching with Hardware" course with more participants. By repeating the experiment described in this paper with multiple teams for each brainstorming method, we hope to confirm or refute our assumptions. It would also be interesting to compare rigid objects to flexible ones.

We plan to use a 3D printer and scanner in order to jump quickly from general ideation to rapid form prototyping. The incorporation of $3 \mathrm{D}$ printing also provides the possibility to fabricate parts that can be combined with parts made of different materials in a modular manner. With this equipment, students could easily scan their selected prototyping forms and offer a variation of their designs in an early usability test setup. We want to know if the general ideation process can be combined with rapid form prototyping or where we have to separate and split the phases. We are aware that these objects also offer some constraints and, at the same time, they can spark an idea.

In subsequent setups, we will try to find how big the balance is between this restriction and a good extension of a process method. At the conference, we want to initiate a discussion based on our results and address the question: Is there a demand for new tools in the user-centered design process as we are moving towards interfaces that might require different ideation techniques to express and develop ideas more easily?

\section{ACKNOWLEDGMENTS}

We would like to thank the students of the course "Sketching with Hardware 09" for their enthusiasm and open feedback. This research has been conducted in the context of the research project PREMIUM and was funded by the DFG (German Research Association).

\section{REFERENCES}

[1] Akaoka, E., \& Ginn, T. \& Vertegaal R.,: DisplayObjects: Prototyping Functional Physical Interfaces on 3D Styrofoam, Paper or Cardboard Models, In Proc. TEI, 2010.

[2] Austin, J.H.: Chase, chance, and creativity: The lucky art of novelty, MIT Press, 2003.

[3] Buchenau, M. \& Suri J.: Experience Prototyping, In Proc. 3rd Conference on Designing Interactive Systems, 2000

[4] Cropley, A.J.: Creativity in education \& learning: A guide for teachers and educators, RouthledgeFalmer, 2001.

[5] Klemmer, S., \& Ju, W., \& Verplank, W., : Teaching embodied interaction design practice In Proc. Conference on Designing for User eXperience, 2005.

[6] Klemmer, S., \& Li,J., \& Lin, J., \& Landay, J., : Papier-Mâché: Toolkit Support for Tangible Input, In Proc. CHI 2004

[7] Mellis, D. \& Banzi, M.: Arduino: An Open Electronic Prototyping Platform, In Proc. CHI, 2007.

[8] Streiz, N. \& Geissler, J.: i-LAND: An interactive landscape for creativity and innovation, In Proc. $\mathrm{CHI}^{\prime} 99$.

[9] Sutton, R., Hargadon, A.,: Brainstorming groups in context: Effectiveness in a product design firm, Administrative Science Quarterly, 1996.

[10] Svanæs, D., \& Seland, G.: Putting the Users Center Stage: Role Playing and Low-Fi Prototyping Enable End Users to Design Mobile Systems, In Proc. CHI, 2004. 